\title{
Evaluating the German Biobank Node as Coordinating Institution of the German Biobank Alliance: Engaging with Stakeholders via Survey Research
}

\author{
Corinna Klingler,,2 Magdaléna von Jagwitz-Biegnitz, ${ }^{1,2}$ Mara Lena Hartung,2 \\ Michael Hummel, ${ }^{2,3}$ and Cornelia Specht ${ }^{2}$
}

Background: The German Biobank Node (GBN) is the central hub for German biobank activities and coordinates the German Biobank Alliance (GBA) to which 11 biobank sites in Germany belong. GBN, in cooperation with designated members of GBA, has developed various services and products for the German biobank community. To ensure that services and products are well aligned with the needs and interests of relevant stakeholders, GBN actively engages with its diverse stakeholder groups through different methods. Important stakeholder groups are the members of GBA who are generally the first users of developed products and services.

Methods and Materials: Members of GBA were surveyed anonymously through a web-based application. The survey was sent to all members registered with an internal communication platform. Participants were primarily asked about their experiences with GBN, developed products and services, their wishes for the future of GBN/GBA, and their attitudes toward intensified cooperation on the European level. Answers were analyzed using descriptive statistics and qualitative content analysis.

Results: Overall 63 of 110 registered GBA members (response rate of 57\%) finished the survey. Participants were overall satisfied with the work of GBN and developed products and services. They also pointed out room for improvement. Participants, for example, proposed to shorten the survey developed for biobanks to engage with their users. They also shared what additional support wishes they had, for example, uniform cost models or use and access policies for the GBA community.

Discussion: The survey was helpful to get a good overview of the experiences and attitudes of GBA members before making services and products available to other actors in the German biobank community. GBN will use the feedback to improve its work and to guide future strategy development. Survey research has shown an adequate method to engage with this particular stakeholder group, but further research on choosing methods for stakeholder engagement might be helpful.

Keywords: German Biobank Node, German Biobank Alliance, stakeholder engagement, user survey, evaluation

\section{Introduction}

\section{The German Biobank Node and Alliance}

F OR Medical Research, project- or department-specific resource. It has been recognized, however, that there are many drawbacks associated with individual or departmental managing of biospecimen collections that are related to quality of samples, ethical handling of data, and sustainability of collections. ${ }^{1}$ Centralized biobanks working under well-defined and controlled conditions address these issues and have accordingly been established increasingly by many research institutions becoming aware of their potential. ${ }^{1}$ This development can also be observed in Germany where many centralized biobanks have been established in the past decade. ${ }^{2,3}$

The growing number of centralized biobanks in Germany made further coordination and harmonization among them necessary. Accordingly, the German Federal Ministry of Research and Education (BMBF) started funding the German

\footnotetext{
${ }^{1}$ QUEST Center for Transforming Biomedical Research, Berlin Institute of Health, Charité Universitätsmedizin Berlin, Berlin, Germany.

${ }^{2}$ German Biobank Node, Charité Universitätsmedizin Berlin, Berlin, Germany.

${ }^{3}$ Central Biobank Charité (ZeBanC), Institute of Pathology, Charité Universitätsmedizin Berlin, Berlin, Germany.
} 
Biobank Node (GBN) as a central hub for German biobank activities in $2013 .{ }^{4}$ GBN also represents the German biobank community in the pan-European network "Biobanking and Biomolecular Resources Research Infrastructure-European Research Infrastructure Consortium" (BBMRI-ERIC). ${ }^{5}$ After a successful first funding period in which concepts for a national biobanking network were developed, a second funding period was granted to develop the network further, build a common IT infrastructure, and establish harmonized quality standards. ${ }^{4}$ The German Biobank Alliance (GBA) coordinated by the GBN was born, consisting of 11 biobank sites (13 biobanks in total) and two IT development centers.*

In close cooperation with designated members of the GBA, the GBN has generated various services and products to support the work of biobanks in Germany. On the GBN website, biobankers can browse the range of products and services already available, including a satisfaction survey for users of biobank services, an image database for demonstrating biobank operations, a manual for quality management and more..$^{\dagger}$ Other available services like ring trial concepts for quality control and training modules for technical biobank personnel are so far mainly available within GBA, but will be made available to other German biobanks in the future.

\section{Aligning activities with needs of stakeholders}

Early on, the GBN emphasized the importance of stakeholder engagement in the development of their products and services. The benefit of engaging with stakeholders has been highlighted for various contexts, particularly health-related research. ${ }^{6-8}$ It has been argued that engaging different stakeholders like patients, clinicians, or policy-makers can not only enhance the quality and relevance of research, but also that it has an intrinsic value to engage those affected, thereby increasing the legitimacy of decisions taken. ${ }^{9,10} \mathrm{In}$ the biobank context, it has also been argued that a strong involvement of stakeholders and consideration of their attitudes and needs will be a prerequisite for implementation of a biobank infrastructure that is actually being used. ${ }^{11,12}$ The GBA community is convinced that the same holds true for a coordinating platform like GBN whose success depends on tailoring its work to the needs and expectations of its stakeholders.

There are different stakeholder groups whose support has to be ensured to guarantee GBN/GBA's success, including researchers, clinicians, patients, the pharmaceutical/diagnostic industry, and the biobanking community itself. To identify the needs of its various stakeholder groups, GBN/GBA have developed a series of activities. Among other things, ${ }^{13}$ a qualitative interview study with researchers using biosamples regarding their attitudes toward centralized biobanks, ${ }^{14}$ a survey with technical personnel working in biobanks to inform the development of a training module, ${ }^{15}$ and a patient survey as basis for a patient campaign ${ }^{16}$ were conducted.

*For an overview of members of the GBA visit our website: https://www.bbmri.de/about-gbn/german-biobank-alliance/?L=1 (visited last on August 28, 2019).

${ }^{\dagger}$ See the following link for an overview of GBN/GBA products made publicly available: http://www.bbmri.de/service/?L=1 (visited last on August 28, 2019).
The most important platform for interaction between GBN and the broader national biobank community is the annual National Biobank Symposium with $>250$ participants. Since the beginning of the project, GBN has used the Symposium to present various developments, to actively promote its products, and to consider the participants' feedback accordingly.

The GBA biobanks are an important stakeholder group of the GBN. They are generally the first to use and employ products and services of GBN. Most products are developed in close cooperation with designated members of GBA, and GBN organizes regular meetings to discuss its work with directors of GBA biobanks. However, other actors having to work with GBN products (like quality managers or technical personnel) have only limited opportunities to share their experiences. At the same time, they are crucial for the success of GBN/GBA and their experiences will also be helpful to improve products and services before their roll-out to other German biobanks. Accordingly, we planned and conducted a survey to get a more well-rounded picture of the perspectives of GBA members. The insights generated thereby will allow GBN to align future strategies with the needs of this important stakeholder group.

\section{Materials and Methods}

No consolidated guidance on reporting of survey research has been published, ${ }^{17}$ but in presenting our findings we have taken different reporting advice into account. ${ }^{18,19}$

\section{Study design and tool}

To get a good idea of how our internal stakeholders perceived the work of GBN/GBA, we decided to conduct a web-based survey. This approach promised to provide a representative picture of satisfaction within GBA. We were also hoping that the anonymity of a web-based approach would provide a context where all could voice their opinion freely without the fear of social repercussions.

In designing our questionnaire, we could not build on established tools owing to the particular questions we were raising. We therefore had to develop a questionnaire from scratch that addresses various aspects of our work: coordination function, developed products and services, expectations for the future, and attitudes toward the BBMRI network and further options for cooperation. For reasons of word count, we did not present all questions included in the survey, but the majority are included. We focused on the questions we perceived as most interesting to the readership of this Journal.

The survey is designed to continuously adjust the questions based on previous answers to reflect the experiences of participants. For example, we asked participants to describe their position in the biobank. As we knew that only quality managers (but not, for example, IT coordinators) were involved in implementation of ring trials, only those were then surveyed on their experiences in this regard. Accordingly, the length of the survey differed. Participants received at least 22 (plus informed consent) and at most 51 (plus informed consent) questions. The 51 questions consisted of 19

\footnotetext{
${ }^{7}$ Further information on the National Biobank Symposium 2019 can be found here: https://www.tmf-ev.de/Termine/ctl/Details/Mid/ 785/ItemID/1512.aspx (visited last on August 28, 2019).
} 
open, 30 single-choice (of which 15 made use of a rating scale) and two multiple-choice questions.

For ethics reasons and to decrease attrition rates, all questions of the survey (except those determining follow-up questions) were voluntary. We used the software SurveyMonkey to technically implement the survey. In developing the survey we were assisted by an established methods specialists who checked the survey for methodological quality $^{20}$ and technical functionality. We also pretested the survey using probing interviews. ${ }^{21}$ Four participants from GBN and the local biobank presenting different areas of expertise were interviewed, their answers analyzed, and the survey adapted accordingly. We have made the survey available in German (the language in which the survey was originally conducted) and English (to make it accessible to an international readership) through the following links:

- https://lamapoll.de/Produkte_Zusammenarbeit_und_ Vernetzung_in_der_GBA (German)

- https://lamapoll.de/Products_cooperation_and_networking_ within_GBA (English). ${ }^{\S}$

\section{Data collection and participants}

We were interested in the opinions of all those engaged with GBN/GBA to which 13 biobanks and 2 IT development centers pertain. We operationalized these to be actors registered at our GBA-internal online communication platform ("Confluence"). All relevant information and updates regarding GBN/GBA are made available on Confluence and it is used to organize collaborative work (e.g., organization of workshops). Access to Confluence has to be granted through the GBN office and requested through directors of GBA biobanks.

We did not draw a sample from those, but sent the survey to all eligible participants $(n=110)$. We sent a first invitation email from the official GBN email account in May 2018. The participation period was communicated to be 3.5 weeks; reminders were sent after 2.5 and 3.5 weeks. No incentives were offered. We did not close the survey until August 2018 when our contract with SurveyMonkey ended. We received two responses 3 and 6 weeks after the announced deadline; all the other responses were provided within the communicated timeline.

\section{Data analysis}

We only conducted descriptive statistical analysis providing an overview of responses. Most of our questions were using a seven-point rating scale with only the lowest and highest scores specifically defined. To each score we assigned a number $(1=$ "Disagree entirely" to $7=$ "Agree entirely") for calculation of the arithmetic mean and standard deviation (SD). The possibility to answer, "I do not know" was rarely offered, but excluded in calculations of the mean and SD were ticked. The open-ended questions of the survey were analyzed using qualitative content analysis by M.J.B. and C.K. ${ }^{22}$ Data were analyzed using the Mi-

\footnotetext{
${ }^{\S}$ As we have no longer access to the software we originally used (SurveyMonkey), the survey has been rebuilt in another software LamaPoll (www.lamapoll.de). While this does not change the content of the survey, it slightly changes its looks.
}

crosoft applications Excel (quantitative analysis) and Word (qualitative analysis).

For reasons of data protection, we did not use cookies or IP addresses allowing to track and attribute responses to individuals. Accordingly, people who did not finish the survey in the first session had to start all over again. Assuming that this might have happened to some people and to avoid counting the answers of participants double, we only included the answers of those that completed the survey (i.e., who arrived at the last page of the survey). However, as most questions were voluntary, we did not exclude anybody for not answering all questions. No further correction method was applied to the data set.

\section{Ethics and data protection}

The study was approved by the ethics commission of Charité Universitätsmedizin Berlin (application number: EA1/071/18). We found that data protection was particularly important in a context where participants assess their colleagues' work. Accordingly, to guarantee anonymity, no personal data that could have allowed identification of participants (including IP addresses) were collected by us.

People were asked to provide informed consent to participate on the first page of the survey by ticking a box. On this page we provided the most important information about the study like purpose and data handling-including that no identifying information about them was being collected. More detailed information was provided in an accompanying pdf document.

\section{Results}

In total, 84 of 110 approached "Confluence" users started filling out the survey, but only 63 completed the questionnaire (yielding a response rate of $\sim 57 \%$ ).

Participants self-identified as quality managers $(n=17)$, biobank managers/project coordinators $(n=17)$, biobank directors $(n=11)$, IT coordinators and coworkers $(n=15)$, technical personnel $(n=9)$, and others including financial and communication specialists $(n=8)$. Typically_particularly in smaller biobanks - employees fill more than one position; participants were therefore given the opportunity to check more than one box describing their role.

\section{Coordination and communication by $G B N$}

We first asked participants to evaluate the work of GBN as the coordinating office in general. Of all participants only $47(75 \%)$ had already been in contact with the GBN office. Accordingly, only those were asked to provide feedback in this regard.

The work of the GBN office was overall positively evaluated (Fig. $1^{* *}$ ). Most of the participants agreed strongly that $\mathrm{GBN}$ is a reliable partner overall (mean $=6.57$; $\mathrm{SD}=0.71)$. They also agreed strongly that $\mathrm{GBN}$ processes enquiries promptly (mean $=6.48 ; \mathrm{SD}=0.74)$, offers wellorganized events $($ mean $=6.26 ; \mathrm{SD}=0.85)$, and provides comprehensive information on relevant GBA activities

\footnotetext{
**We provide figures to accompany narrative data presentation only exemplarily.
} 
The GBN office...

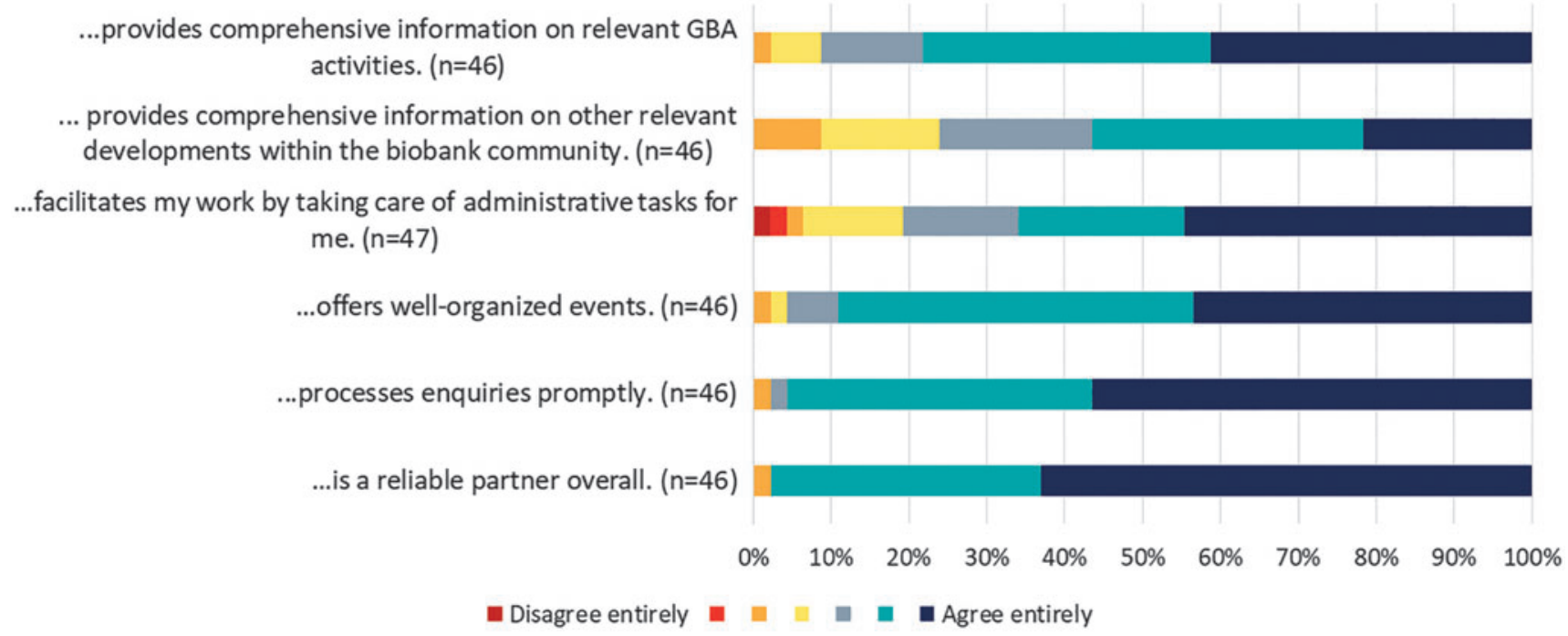

FIG. 1. Perspectives on the work of the GBN office. GBN, German Biobank Node.

(mean $=6.09 ; \mathrm{SD}=1.00)$. They also agreed (but less strongly) that GBN facilitates the work within GBA by taking care of administrative tasks (mean $=5.79 ; \mathrm{SD}=1.46)$ and provides comprehensive information on relevant developments within the biobank community (mean $=5.46 ; \mathrm{SD}=1.23$ ).

Participants furthermore strongly agreed that the GBN team is friendly (mean $=6.83 ; \mathrm{SD}=0.52)$, competent $($ mean $=6.57$; $\mathrm{SD}=0.77)$, helpful $($ mean $=6.8 ; \mathrm{SD}=0.54)$, and does a good job overall $($ mean $=6.67 ; \mathrm{SD}=0.66)$.

\section{Communication tools developed by GBN}

GBN employs different tools to communicate with its stakeholders. One communication tool employed is the $G B N$ website where developments within GBA or BBMRI-ERIC are reported, further developments of relevance to the community discussed and new products, publications, and events shared. In October 2017, the GBN website in its current format was launched. Fifty participants (79\%) were aware of the relaunched GBN website, of which $84 \%(n=42)$ use the GBN website also as information source. In cases where participants stated that they did not use the website, they identified in an open question most often the lack of time and alternative information sources as reasons for neglecting the website.

Those using the website as an information source agreed that the website has an appealing design (mean $=6.10$; $\mathrm{SD}=1.03)$ and is clearly structured (mean $=5.85 ; \mathrm{SD}=1.05$ ), easy to navigate (mean $=5.85 ; \mathrm{SD}=1.16$ ), well written (mean $=6.02 ; \mathrm{SD}=1.05)$, and features interesting content $($ mean $=5.61 ; \mathrm{SD}=1.21)$. With regard to opportunities for improvement (open question), participants commented that they would like publications to be sorted by topics and not years, to include more (and improve accessibility of) information on public relations and did not always found the labels used for sorting information helpful.

Another tool for communication is the GBN newsletter. It targets internal and external stakeholders of GBN and contains information on current developments within the biobank community and interviews with diverse experts in the field. The newsletter in its current format was launched in 2018; two had been sent out (through e-mail) at the time of surveying. Of 49 participants who already knew the newsletter in its new format, 46 had read at least one GBN newsletter. They found the newsletter to be well written (mean $=5.91$; $\mathrm{SD}=0.95)$, clearly structured (mean $=5.78 ; \mathrm{SD}=1.00)$, to have an appealing design (mean $=5.72 ; \mathrm{SD}=0.99)$, and feature interesting content (mean $=5.50 ; \mathrm{SD}=1.02)$. Of the participants who provided feedback on improvement opportunities (open question), one would have liked the newsletter to be shorter and the other would have liked the newsletter to contain whole articles instead of teasers with links to full texts.

Those not reading the newsletter were given the opportunity to share their reason. They answered to the open question that they never read newsletters, did not read ours for lack of time, only read parts of it (and were missing this option in the survey), or were sufficiently informed by other sources (e.g., personal meetings and Confluence updates).

Furthermore, the GBN had developed a poster campaign as part of the donor communication strategy. Posters are supposed to be displayed at each GBA biobank site and are offered in four different designs (Fig. 2 e.g.). Distribution to GBA biobanks has started in June 2018. Local quality managers or project coordinators are responsible for distribution locally and were accordingly the only ones given questions regarding the poster campaign.

Participants strongly supported the poster campaign (Fig. 3). They found the developed posters to be appealing (mean $=6.21 ; \mathrm{SD}=0.92$ ) and posters to be a good strategy to increase awareness for biobanks among potential donors of biosamples (mean $=6.38 ; \mathrm{SD}=0.76)$. All participants plan to implement the poster campaign at their institution (mean $=6.39 ; \mathrm{SD}=0.82$ ). Participants responded to an open question that the campaign would benefit from additional support like videos and social media material, but one participant also explicitly stated that the available support is sufficient. 


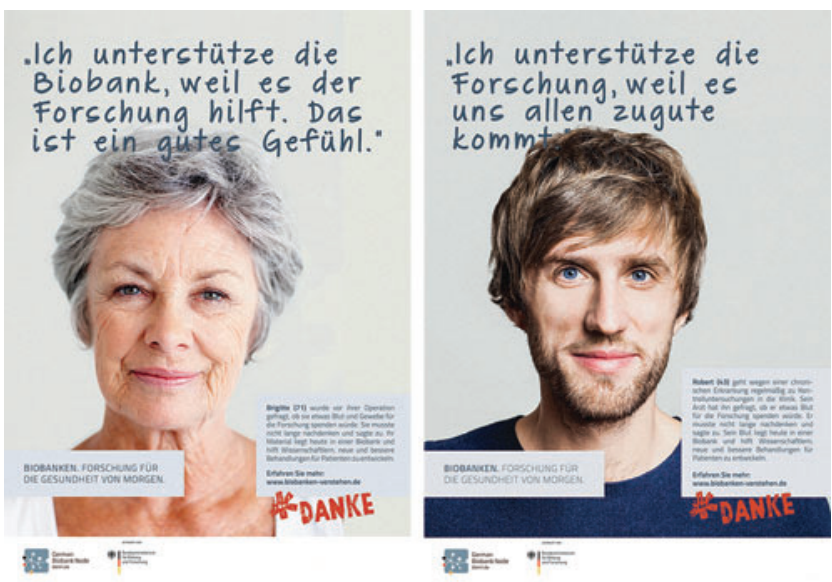

FIG. 2. Examples of posters from the campaign targeting sample donors. English translation of main text of Poster 1: I am supporting the biobank because it helps research. It's a good feeling. English translation of main text of Poster 2: I am supporting research because it benefits us all. The smaller boxes explain the story of why people on the posters decided to donate biosamples to the biobank.

Where biobanks do not cater to the needs of their users, they will likely not be successful. Therefore, GBN developed a questionnaire to be used by the GBA biobanks. This satisfaction survey was targeting users of biobank services (i.e., researchers) and was meant to enable the GBA biobanks to communicate with their users about their preferences, needs, and experiences with the respective biobank. We were interested whether the questionnaire actually helped the biobanks in attaining relevant feedback.

The great majority of targeted participants $(n=29)$ strongly supported implementation of the user satisfaction survey $($ mean $=6.88 ; \mathrm{SD}=0.32$ ) and found it helpful to improve internal processes $($ mean $=6.26 ; \mathrm{SD}=1.14)$. Although four participants were unsure, most are using or planning to use the results of the survey to improve their offers $($ mean $=6.30 ; \mathrm{SD}=0.86)$. Responses to the open question regarding suggestions for improvement showed that the questionnaire was perceived as too long (with lengths being identified as reason for low response rates to survey). Furthermore, the presentation of findings was considered suboptimal for easy interpretation.

\section{Quality management tools}

GBN, in cooperation with selected members of GBA, developed several tools to improve the quality of services provided by the biobanks, including a quality management $(Q M)$ manual. It was created during the previous funding period and is based on different quality standards like DIN EN ISO 9001:2015, 17025, 17020 and 15189. It combines the established standard operating procedures (SOPs) biobanks already use and the outcome of various GBA quality management workshops. In its final form, the manual contains template documents, biobank-specific SOPs, and a self-assessment questionnaire regarding the existing QM system of a biobank.

The questions about the manual were addressed exclusively to the group of quality managers $(n=17)$. Fifteen quality managers confirmed that the manual is used in their biobank. Participants generally agree that the QM manual is clearly structured (mean $=5.57 ; \mathrm{SD}=0.98$ ) with content of high quality $($ mean $=5.93 ; \mathrm{SD}=0.96)$ and practical orientation $($ mean $=5.46$; $\mathrm{SD}=1.39)$. Whether the manual facilitates the work of quality managers was seen as more critical (mean $=4.92 ; \mathrm{SD}=1.59$ ) with two participants disagreeing (one strongly) with the statement "The QM manual facilitates my work." Open questions showed that people found it difficult to adapt generic templates and SOPs to their own situation.

Finally, two ring trials (one focusing on tissue and another on liquid biobanking) were implemented to compare quality across GBA biobanks. The tissue ring trial (Fig. 4) was seen rather positively with participants showing strong agreement with statements like, the ring trials was well organized $($ mean $=5.86 ; \mathrm{SD}=1.25)$, detailed procedure for the ring trial was communicated in advance (mean $=5.93$; $\mathrm{SD}=1.39$ ), the trial could be implemented at the site without any issues $($ mean $=5.71 ; \mathrm{SD}=1.28)$, and outcomes will help me to review on-site processes and improve these as necessary $($ mean $=6.14 ; \mathrm{SD}=0.99)$.
To what extent do you agree with the following statements about the poster campaign?
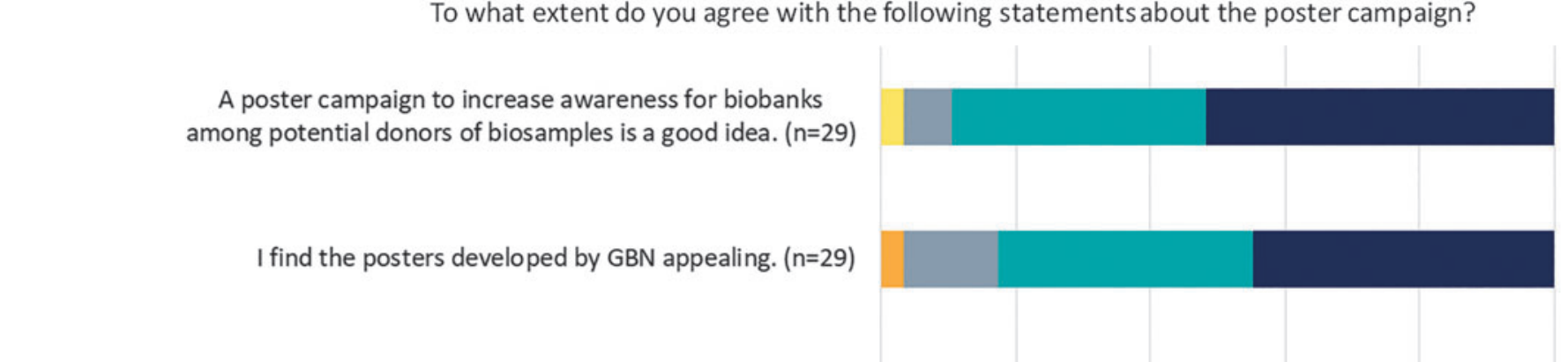

A poster campaign to increase awareness for biobanks among potential donors of biosamples is a good idea. $(n=29)$

I find the posters developed by GBN appealing. $(n=29)$

I plan to implement the poster campaign at my biobank. $(n=28)$ 


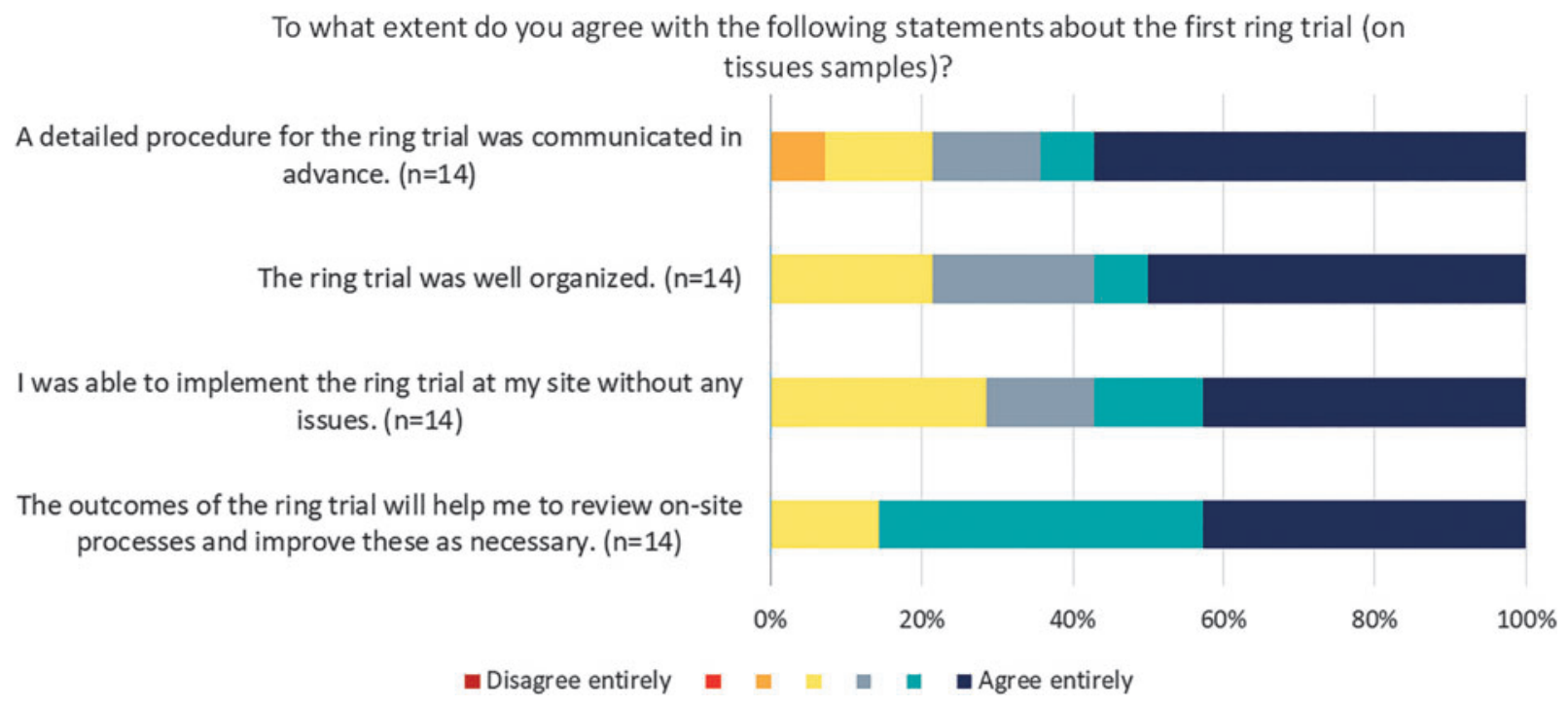

FIG. 4. Perspectives on the tissue ring trial.

The liquid ring trial (Fig. 5) was seen more critically with less agreement on the following statements: the ring trials was well organized (mean $=4.29 ; \mathrm{SD}=1.56$ ), detailed procedure for the ring trial was communicated in advance ( mean $=4.65 ; \mathrm{SD}=1.91)$, the trial could be implemented at the site without any issues (mean $=5.5 ; \mathrm{SD}=1.06)$, and outcomes will help me to review on-site processes and improve these as necessary (mean $=5.2 ; \mathrm{SD}=1.83$ ).

Among the points criticized in responses to an open question for improvement opportunities were inadequate timing, as another GBA event for technical personnel (which was needed to conduct the analyses) was scheduled for the same day and frequent change of plans making adequate preparation difficult.

\section{Wishes for future product and service development}

We also asked an open question regarding wishes for future product and service development. Answers were diverse and ranged from image campaigns to acquisition of a common QM software. Table 1 presents the coding system generated from answers provided.

\section{International cooperation within BBMRI-ERIC}

Of all 63 participants, $46(73 \%)$ stated that they are interested in intensifying networking on the European level within BBMRI-ERIC. Those interested in further collaboration were asked about their expectations regarding the European network. Table 2 presents the coding system generated from answers provided to this open question.

Using a multiple-choice question, we also asked in which of BBMRI-ERIC's areas of activity they would be prepared to get more involved. The participants showed interest in becoming more involved in quality management $(n=17)$, IT $(n=13)$, stakeholder management $(n=8)$, and ethical, legal, and social issues $(n=4)$. Five participants stated their interest to be involved in "other areas of activity," which they specified as training and education, strategy and finance, development of a biobank/business plan, and the topic of

To what extent do you agree with the following statements about the second ring trial (on liquid samples)?

A detailed procedure for the ring trial was communicated in advance. $(n=17)$

The ring trial was well organized. $(n=17)$

I was able to implement the ring trial at my site without any issues. $(n=16)$

The outcomes of the ring trial will help me to review on-site processes and improve these as necessary. $(n=15)$

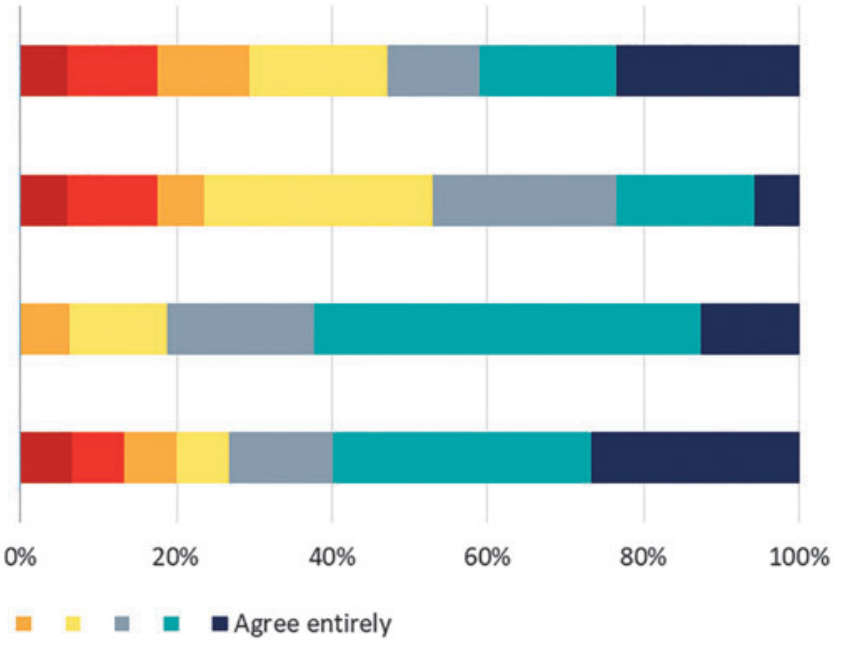

- Disagree entirely

FIG. 5. Perspectives on the liquid ring trial. 
Table 1. Coding System Presenting the Wishes for Future Service and Product Development

Development of further
products or improvements
in the area of QM

Central QM software

Templates for service contracts with diverse customers

Templates for interface agreements with external service providers

Internal audits

Better coordination of future biomarker ring trials

Development of approaches for process harmonization

Uniform cost models

Ensuring the acceptance of cost models by potential sponsors

Uniform Use and Access Policies

Uniform Material/Data Transfer Agreements (developed jointly with Medical Informatics Initiative)

Organization of further events Further training courses for biobank employees

Training opportunities for staff outside of biobanks

Regular meetings of all German biobanks to promote exchange

Improve public image through

GBA should act as collective

Press campaign

Listing of the service offered by GBA biobanks

bargaining partner to

achieve lower prices from suppliers

GBA, German Biobank Alliance; QM, quality management.

rare diseases. Nine participants stated that they did not want to get more involved in BBMRI-ERIC's areas of work.

Seventeen participants stated to have no interest in intensifying networking efforts on the European level. They were given the opportunity to elaborate on their reasons in answers to an open question. Four main reasons were identified: lack of time and resources, local focus, an effective national network in Germany as a priority (for now), and already sufficient networking at European level.

\section{Discussion}

The survey has shown that participants were in general content with the work, services, and products of GBN/GBA. This is reassuring feedback before rolling out the products/services to the rest of the German biobank community. People have also pointed out where room for improvement exists. This feedback has been analyzed and discussed within GBN and steps have been and will be taken to adapt our services accordingly.

However, feedback will not uncritically be translated into action as sometimes feedback is inconsistent, for example, where the newsletter is considered too long by some and not providing enough information by others, we considered the current format to be an acceptable compromise. Another reason is that other information sources might contradict or at least put

Table 2. Coding System Presenting THE Expectations of European Networking

Exchange of experiences
and knowledge transfer

Cooperation with actors outside Germany

Facilitation of EU-wide exchange of samples and data

Alignment with interests of international stakeholders

More visibility and better positioning of biobank community

EU-wide harmonization of processes to ensure high standards of practice

Realization of synergies/reduction of national work load

Access to funding

Compatibility of IT used

certain answers in perspective. The GBN newsletter, to stick to the example, is well received by comparison: its open rate is significantly above average. ${ }^{\dagger \dagger}$ Some proposals are unfortunately unfeasible, for example, GBN is lacking the necessary funds to produce videos for improved donor engagement.

Some feedback has already been considered in the revision of our processes and services. For example, we bought and distributed a common QM software as desired by participants that will also facilitate implementation of the QM manual in the daily work routines of quality managers and support the internal audits. We have also revised our user satisfaction survey, which has been shortened and is now

\footnotetext{
${ }^{\dagger}$ The email marketing provider Newsletter2Go published a study which compares newsletter click and open rates. They analyzed 360 million emails from 29 different industries sent via Newsletter2Go from 30 June 2017 to 30 June 2018, see: https://www.newsletter2go .de/whitepaper/branchen-benchmark-2018/(last visited on August $28,2019)$. In it, they calculate a cross-industry average open rate of $26,56 \%$. With $50,64 \%$ the open rate of the GBN newsletter was almost twice as high in 2018 (the first edition of the GBN newsletter was published in February 2018, analysis conducted with Mailchimp reports).
} 
realized in a format that is more easily accessible. We are also working on offering additional support in accordance with wishes raised by our stakeholders. So far, we have (1) developed an internal audit program, which is now being implemented throughout GBA biobanks and (2) organized several workshops to provide an introduction to existing cost models and to develop a uniform model. In addition, external training courses and webinars offered by other European partners are better promoted and the exchange at the national annual meetings and the Europe Biobank Week is supported.

In the future, we will address further points that have been raised, such as providing templates for contractual agreements. We will also pass relevant information on to BBMRI-ERIC.

We successfully used survey research to engage with the perspectives of one relevant stakeholder group. Other methods for engagement like round tables, representing relevant stakeholders on advisory boards, focus groups, interviews, or deliberative democracy events have also been successfully used in the biobank context. ${ }^{23-25}$ Which method is most adequate will depend on the specific goal and targeted stakeholder group? Based on the positive experience with surveying GBA members, we are planning to re-evaluate our products and services toward the end of the current funding period. We will then integrate all national biobanks in the survey to measure the outreach of the activities of GBN/GBA to the biobanking community.

\section{Limitations}

We did not use IP addresses or Cookies to track individual responses, so people could have filled out the survey twice or pass the survey link on to those not eligible for participation. However, there is little reason to suspect people to do so as they themselves have an interest in sound results. We have also not added any cross-check questions to the survey as we did not want to increase its already considerable length and thereby discourage people from participating. We also found it less relevant in this context as we considered the risk of misrepresentation or misunderstanding to be low which was confirmed in the pilot test.

In addition, we have a moderate response rate of 57\%, which is even lower for some of the nonmandatory questions. However, response rates of $>50 \%$ are generally considered acceptable. ${ }^{26}$ Furthermore, all important functions within GBA (biobank directors and managers, IT coordinators and quality managers) have been adequately represented among the sample. We therefore believe that the results should be considered robust.

However, as we did not ask why people decided not to participate, we cannot be sure whether any important differences among responders and nonresponders existed. We can only speculate that the lengths of the survey might have discouraged some GBA members. On average, it took participants 29 minutes from opening to completion of the survey with a median time of 16 minutes. " In addition,

\footnotetext{
Hudging from the excessive time spend on the survey (more than seven hours for one participants), some participants probably did other things in between. Accordingly, it is not clear how long participants actually spend on filling out the survey. The median time, however, might be more informative in this regard than the mean.
}

others that are only involved with GBN/GBA in specific projects might have felt insufficiently knowledgeable about the work of GBN/GBA to take the survey. This might be particularly true for technical personnel.

\section{Concluding remarks}

Stakeholder engagement is important to adapt services and products to the needs and wishes of relevant stakeholders. Products and services of GBN/GBA have been positively evaluated by employees of GBA biobanks in this survey, but areas for improvement have also been identified. Further engagement activities are planned to receive feedback from biobank employees outside of the GBA network. Although the survey provided us with relevant feedback, further research analyzing which engagement methods are most effective in which context and for which stakeholder group might be very helpful for the future.

\section{Acknowledgments}

The authors thank Christiane Hartfeldt, Ida Steier, Cäcilia Engels, and Dana Briesemeister for helping us in pilot testing the survey. We are indebted to all participants for investing their time to provide us with the necessary feedback and the Federal Ministry of Education and Research for funding the GBA and thereby this study.

\section{Author Disclosure Statement}

The authors declare that they all are or have been working for the institution being evaluated in this study.

\section{Funding Information}

This study was funded by the Federal Ministry of Education and Research (BMBF) of Germany as part of the broader project "Deutsche Biobank Allianz/BBMRI.de Nationaler Knoten" (funding code 01EY1701).

\section{References}

1. Larsson A. The Need for Research Infrastructures: A Narrative Review of Large-Scale Research Infrastructures in Biobanking. Biopreserv Biobank 2017;15:375-383.

2. Pigeot I, Hummel M. Biobanken-eine entscheidende Ressource für erfolgreiche biomedizinische Forschung. Bundesgesundheitsblatt 2016;59:301-303.

3. Bernemann I, Kersting M, Prokein J, et al. Zentralisierte Biobanken als Grundlage für die medizinische Forschung. Bundesgesundheitsblatt 2016;59:336-343.

4. Bundesministerium für Bildung und Forschung. Biobanken - nationale Förderung/BBMRI. Available from: https://www .gesundheitsforschung-bmbf.de/de/nationale-biomaterial banken-initiative.php.

5. Mayrhofer MT, Holub P, Wutte A, et al. BBMRI-ERIC: The novel gateway to biobanks. Bundesgesundheitsblatt 2016;59:379-384.

6. INVOLVE. Briefing Notes for Researchers: Involving the Public in NHS, Public Health and Social Care Research. Eastleigh: INVOLVE; 2012.

7. Geissler J, Ryll B, di Priolo S, et al. Improving Patient Involvement in Medicines Research and Development: A Practical Roadmap. Ther Innov Regul Sci 2017;51:612-619.

8. Frank L, Forsythe L, Ellis L, et al. Conceptual and practical foundations of patient engagement in research at the 
patient-centered outcomes research institute. Qual Life Res 2015;24:1033-1041.

9. Gradinger F, Britten N, Wyatt K, et al. Values associated with public involvement in health and social care research: A narrative review. Health Expect 2015;18:661-675.

10. Hansen SL, Holetzek T, Heyder C, et al. StakeholderBeteiligung in der klinischen Forschung: Eine ethische Analyse. Ethik Medizin 2018;30:289-305.

11. Bjugn R, Casati B. Stakeholder analysis: A useful tool for biobank planning. Biopreserv Biobank 2012;10:239-244.

12. Simeon-Dubach D, Watson P. Biobanking 3.0: Evidence based and customer focused biobanking. Clin Biochem 2014;47:300-308.

13. Schüttler C, Buschhüter N, Döllinger C, et al. Anforderungen an eine standortübergreifende Biobanken-IT-Infrastruktur. Der Pathologe 2018;39:289-296.

14. Schütt A, Lesch W, Jahns R. Was erwarten Wissenschaftler von zentralisierten Biobanken? Eine qualitative Stakeholder-Analyse. In: Lesch W, Schütt A (eds). Gesundheitsforschung kommunizieren, Stakeholder Engagement gestalten: Grundlagen, Praxistipps und Trends. Berlin: Medizinisch Wissenschaftliche Verlagsgesellschaft; 2016: 161-176.

15. Hartung ML, Herpel E, Baber R, et al. German Biobank Alliance: Implementation of an educational program for technical personnel in biobanks. Presented at: European Biobank Week (EBW) 2018, Antwerpen.

16. Lesch W, Schuett A, Herpel E, et al. How well do study participants understand the concept of biobanking in medical research? Results of a survey among sample donors in Germany. Presented at: Conference of the International Society for Biological and Environmental Repositories 2016, Berlin.

17. Bennett C, Khangura S, Brehaut JC, et al. Reporting guidelines for survey research: An analysis of published guidance and reporting practices. PLoS Med 2010;8: e1001069.
18. Eysenbach G. Improving the quality of Web surveys: The Checklist for Reporting Results of Internet E-Surveys (CHERRIES). J Med Internet Res 2004;6:e34

19. Kelley K, Clark B, Brown V, et al. Good practice in the conduct and reporting of survey research. Int J Qual Health Care 2003;15:261-266.

20. Porst R. Fragebogen: Ein Arbeitsbuch. 4 ed. Wiesbaden: Springer VS; 2014.

21. Prüfer P, Rexroth M. Kognitive Interviews. ZUMA How-toReihe No.15. Available at: https://www.ssoar.info/ssoar/ handle/document/20147; 2005.

22. Schreier M. Qualitative Content Analysis in Practice. Los Angeles, London: Sage; 2012.

23. Lemke AA, Wu JT, Waudby C, et al. Community engagement in biobanking: Experiences from the eMERGE Network. Genom Soc Policy 2010;6:50.

24. Mitchell D, Geissler J, Parry-Jones A, et al. Biobanking from the patient perspective. Res Involv Engage 2015;1:4.

25. Baldo C, Casareto L, Renieri A, et al. The alliance between genetic biobanks and patient organisations: The experience of the telethon network of genetic biobanks. Orphanet $\mathbf{J}$ Rare Dis 2016;11:142.

26. Draugalis JR, Coons SJ, Plaza CM. Best practices for survey research reports: A synopsis for authors and reviewers. Am J Pharm Educ 2008;72:11.

Address correspondence to: Dr. Corinna Klingler, $M S c, B A$ German Biobank Node

Charité - Universitätsmedizin Berlin Campus Virchow-Klinikum Augustenburger Platz 1 13353 Berlin Germany

E-mail: corinna.klingler@charite.de 